Fission yeast goes synthetic.

Nielsen, Olaf

Published in:

Nature Methods

DOI:

10.1038/nmeth1007-777

Publication date:

2007

Document version

Early version, also known as pre-print

Citation for published version (APA):

Nielsen, O. (2007). Fission yeast goes synthetic. Nature Methods, 4(10), 777-8.

https://doi.org/10.1038/nmeth1007-777 


\section{Fission yeast goes synthetic}

\author{
Olaf Nielsen
}

\section{A high-throughput procedure for genome-wide identification of genetic interactions in Schizosaccharomyces pombe.}

Genetic analysis is probably our most powerful method for obtaining mechanistic insight into cellular function. The geneticist uses a reverse 'destructive' logic: by mutating a particular gene and studying the resulting phenotype, the function of that gene can be inferred. This type of analysis is particularly well suited for genes that perform a unique function in the cell. But if the genome contains a second gene encoding a related or redundant function, this can mask the phenotype of the mutant. In this issue of Nature Methods, Krogan and colleagues describes a protocol for systematic identification of genetic interactions between gene pairs in the fission yeast $S$. pombe.

Systematic gene-deletion studies in budding yeast have revealed that a relatively large class of genes can be eliminated without any apparent phenotypic consequences for the cell. Several different explanations can account for this. First, some genes only become important under certain circumstances; they are not necessary under the highly artificial conditions used to propagate yeast cells in the laboratory. A fraction of these genes may no longer have a function-something that is very difficult to substantiate in practice-but the majority of genes that can be mutated without an apparent phenotype are likely to be genetically redundant. In such cases, the geneticist can attempt to identify the function by looking for other genes that when mutated confer a phenotype on the first mutant.

When the phenotype of a mutant
Mate query deletion mutant to deletion library
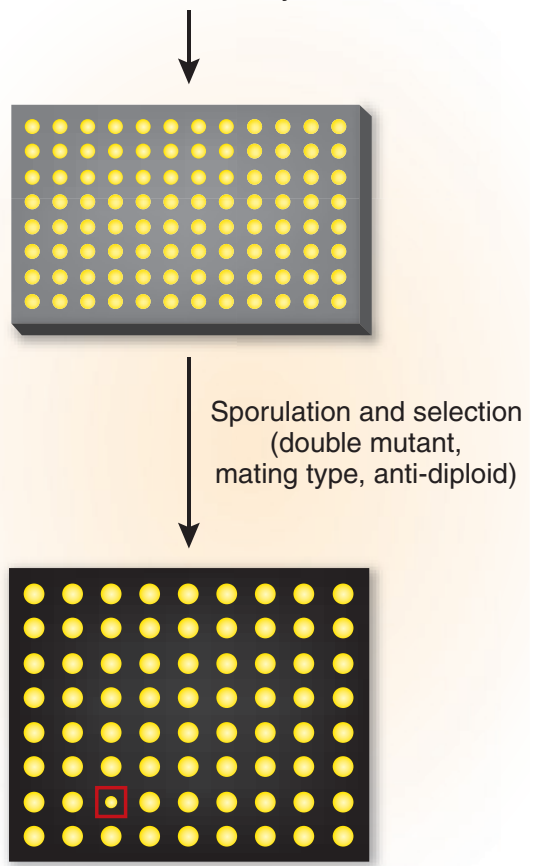

Identification of synthetic lethal interactions

Figure 1 | Screen for genetic interaction between gene pairs in the fission yeast S. pombe. Synthetic lethal interactions are detected as growth inhibition (red square).

depends on the status of a second gene, the two genes are said to interact genetically, and usually this indicates that the two genes function in the same pathway. By analogy, if a person wears suspenders, you would have to cut both shoulder straps to bring down the trousers.

Olaf Nielsen is at the University of Copenhagen, Department of Molecular Biology, Ole Maaløes Vej 5, DK-2200 Copenhagen N, Denmark.

e-mail: onigen@my.molbio.ku.dk

But because the relationship between the two genes is based on phenotypic observations, it is unclear whether the interaction is direct (if the two gene products function in the same biochemical process) or indirect (if the other gene product functions in a compensatory pathway). To continue the above metaphor, if a person wears both suspenders and a belt, both these contraptions need to be inactivated to observe the trousersdown phenotype.

A particularly compelling type of genetic interaction occurs when the combined inactivation of two genes causes cell death. This kind of relation, referred to as a synthetic lethal interaction, indicates that the two genes in combination carry out an essential function in the cell. Again, the relationship can be direct (for example, if two protein kinases can perform the same essential phosphorylation reaction ${ }^{2}$ ) or indirect. For instance, fission yeast cells can tolerate a mutation that slows down DNA replication because of reduced nucleotide pools, but if the (normally unessential) DNA structure checkpoint pathway simultaneously is inactivated, the cells $\mathrm{die}^{3}$. The interpretation of this experiment is that DNA replication in the mutant relies on the function of the checkpoint pathway.

Screens for synthetic lethal interactions in yeast therefore have been instrumental in understanding dependency relationships between gene functions. Initially, such screens were performed by identifying genomic mutations that made cell survival dependent on a plasmid expressing the query gene product ${ }^{4}$. With the advent of systematic deletion programs, it became possible to monitor synthetic lethal relationships on a genome-wide scale $^{5}$. Subsequently, such screens were simplified by replacing plate-based crosses with cell pools and then identifying the non-growing synthetic lethal combinations by hybridization to microarrays that detect unique DNA bar-code sequences present in the deletions ${ }^{6}$.

To systematically identify synthetic interactions in fission yeast, Krogan and 
colleagues developed two protocols that find synthetic lethal interactions between a query mutant strain and a genome-wide deletion library (which is now available, albeit only commercially). Owing to the life cycle of fission yeast, where mating and meiosis are induced under the same physiological conditions, the procedures developed for budding yeast had to be modified in sev응 eral ways, but this difference also allows one of the screens to be completed in only 9 days. Both methods combine a selection for double mutants of a particular mating type with a selection against unsporulated diploid cells. The anti-diploid selection is cleverly based on a newly developed reces- sive marker that confers resistance to the drug cycloheximide.

What can we learn from a screen for synthetic lethal interactions in fission yeast that we did not already know from work in budding yeast? First, it is important to realize that these two yeasts are only very distantly related. Hence, genetic interactions that are conserved between them are more likely to be found in other more complex eukaryotes as well. Furthermore, because of its highly specialized mitotic cell cycle, budding yeast in many respects appears to have evolved away from the prototype eukaryote cell. Fission yeast, in contrast, appears to be less specialized and has retained several key ancient cellular processes (for example, large complex centromere structures containing RNAi- and histone methylation-dependent heterochromatin) that are no longer found in budding yeast. Thus, the entry of $S$. pombe into the era of synthetic lethal screens is bound to produce exciting insights.

1. Roguev, A. et al. Nat. Methods 4, 861-866 (2007).

2. Lundgren, K. et al. Cell 64, 1111-1122 (1991).

3. Holmberg, C. et al. Genes Dev. 19, 853-862 (2005).

4. Bender, A. \& Pringle, J.R. Mol. Cell. Biol. 11, 1295-1305 (1991).

5. Tong, A.H. et al. Science 294, 2364-2368 (2001).

6. Ooi, S.L. et al. Nat. Genet. 35, 277-286 (2003). 